\section{Apomorphin- und Levodopa-Pumpentherapie im Vergleich}

Eine aktuelle, multizentrische Beobachtungsstudie bei Parkinson-Patienten im fortgeschrittenen Krankheitsstadium weist einen robusten und vergleichbar großen Effekt beider Pumpen-Therapieverfahren zur Behandlung motorischer und zum Teil auch nicht motorischer Symptome sowie auf Komplikationen und die Lebensqualität nach.

$\mathrm{N}$ eben der Tiefenhirnstimulation (THS) stellen Pumpentherapien mit Apomorphin subkutan (APO) oder Levodopa intrajejunal (IJLI) die erfolgversprechendsten etablierten Optionen zur nicht oral medikamentösen Behandlung von Patienten mit idiopathischem Parkinsonsyndrom (IPS) und motorischen Komplikationen dar. Im Gegensatz zur breiten Datenlage zur THS war die Evidenz für beide Pumpentherapien bis vor kurzem relativ begrenzt. Zur IJLI-Therapie existiert mittlerweile Klasse-1-Evidenz auf dem Boden einer doppelblinden, randomisierten Studie. Direkte prospektive Vergleichsstudien der beiden Pumpenverfahren fehlten bisher.

Die vorliegende Studie von MartinezMartin et al. [1] stellt die erste prospektive, multizentrische, offene Beobachtungsstudie zum Vergleich von APOund IJLI-Therapie dar. Insgesamt $77 \mathrm{~Pa}$ tienten wurden auf eine der beiden Pumpenverfahren (44 IJLI, 43 APO) eingestellt und wurden nach sechs Monaten nachuntersucht. Einschlusskriterien waren eindeutige Dopa-Responsivität, das Fehlen einer Demenz und ausgeprägte, durch konventionell oral medikamentöse Ansätze nicht zufriedenstellend zu beeinflussende motorische Komplikationen wie Fluktuationen und Dyskinesien. Zielparameter waren die Beeinflussung der Hoehn \& Yahr-Skala, des UPDRS 3 und 4, der Non-Motor Symptom Scale (NMSS) und der Lebensqualität gemessen am Parkinson's Disease Questionnaire (PDQ-8).

Die Zusammensetzung beider Therapiegruppen war hinsichtlich Alter, Krankheitsdauer, Lebensqualität sowie Schweregrad motorischer und nicht motorischer Symptome zu Studienbeginn vergleichbar. Unter Therapie war die mittlere Levodopa-Dosis beziehungsweise Levodopa-Äquivalenz-Dosis in beiden
Gruppen ohne signifikanten Unterschied (2017 mg/Tag IJLI, 1934 mg/Tag APO), ebenso die tägliche Laufzeit der Pumpe (17,3 Stunden IJLI, 15,9 Stunden APO). Alle Zielparameter der Studie hinsichtlich motorischer Symptome (UPDRS3), motorischer Komplikationen (UPDRS4) und Lebensqualitätssummenscore (PDQ-8) besserten sich signifikant und vergleichbar unter beiden Therapien. Der nicht motorische Summenscore (NMSS) sowie Unterpunkte des NMSS wie Schlaf/Müdigkeit, gastrointestinale sowie Sexual- und Blasenfunktion besserten sich stärker unter der IJLI-Therapie. Auf Müdigkeit und Apathie zeigte APO den stärkeren Effekt.

In der APO-Gruppe wurden keine schwerwiegenden Nebenwirkungen beobachtet, allerdings auch schwere Müdigkeit in drei und Impulskontrollstörung bei vier Patienten. In der IJLIGruppe waren Sondenkomplikationen relativ häufig, in einem Fall schwerwiegend im Sinne einer Peritonitis.

\section{Kommentar}

Die vorliegende Studie stellt die bisher größte prospektive Vergleichsstudie von APO- und IJLI-Therapie, zudem in einem multizentrischen Ansatz bei fortgeschrittenem IPS dar. Sie zeigt über einen Zeitraum von sechs Monaten, dass beide Verfahren vergleichbare und durchschlagende Effekte auf motorische Symptome und Lebensqualität der Patienten erzielen. Optimal wäre hier natürlich eine doppelblinde, randomisierte Studie, die aus technischen/logistischen Gründen aber wohl kaum auf absehbare Zeit durchgeführt werden wird. Bedingt durch das Studiendesign und die Größe der beiden Gruppen sind die Ergebnisse sicher als nicht abschließend konklusiv zu betrachten.

Hinsichtlich der Beeinflussung nicht motorischer Symptome (Summenscore und eini-

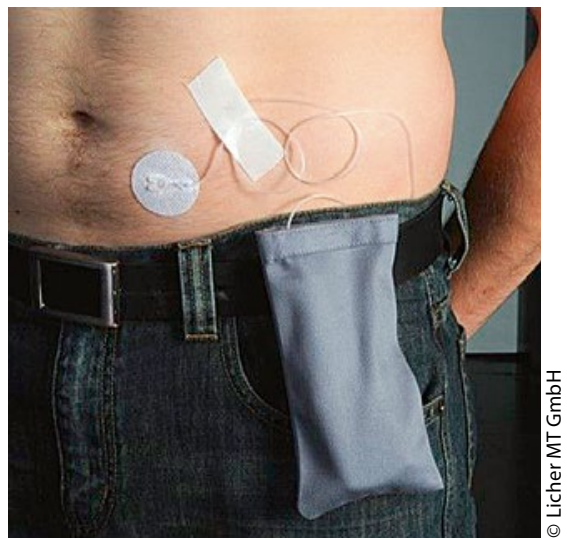

Anlage einer Apomorphin-Pumpe.

ge Unterdomänen) scheint die IJLI-Therapie leichte Vorteile gegenüber der APO-Therapie zu haben, wenngleich dies sicherlich ein eher deskriptives Ergebnis darstellt, was in weiteren kontrollierten Studien bestätigt werden müsste.

Die Komplikationsrate scheint in der IJLIGruppe vor allem bedingt durch Sondenbezogene Probleme höher als unter APO zu sein. In der APO-Gruppe dominieren eher Hautreaktionen wie subkutane Knotenbildung und Tagesmüdigkeit. Letzteres kann aber bei fortgeschrittenen IPS-Patienten ebenfalls problematisch sein. Interessanterweise traten Impulskontrollstörungen auch in der APO-Gruppe nur sehr selten auf, in der IJLI-Gruppe waren sie kein Thema. Insgesamt sollte die Entscheidung zu einem der beiden Pumpenverfahren natürlich immer auf individueller Basis fallen, hier spielt auch die Akzeptanz beim Patienten eine große Rolle. Zu letzterer trägt vor allem die engmaschige ambulante Nachbetreuung der Patienten in Zusammenarbeit mit geschultem Pflegepersonal bei. Insbesondere für die IJLI-Therapie ist die Zusammenarbeit eines spezialisierten geschulten Teams aus Neurologen und Gastroenterologen essenziell. Beide Therapien verlangen gutes Monitoring potenzieller Komplikationen (APO: Haut, nicht motorische Nebenwirkungen; IJLI: Sondenkomplikationen, Neuropathie), stellen hierunter aber eine sichere und effektive Therapieoption für fortgeschrittene Patienten dar.

Prof. Dr. med. Bernhard Haslinger

1. Martinez-Martin P et al. Eurolnf: a multicenter comparative observational study of apomorphine and levodopa infusion in Parkinson's disease. Mov Disord 2015; 30 (4): $510-6$ 